\title{
GRUPO DE ESTUDOS LINGUÍSTICOS DO ESTADO DE SÃO PAULO
}

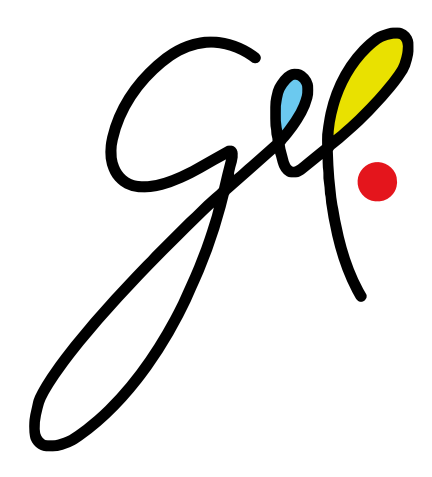

\section{ESTUDOS LINGUÍSTICOS \\ v. 48, n. 2}

ESTUDOS LINGUÍSTICOS, São Paulo, 48 (2): p. 581-1149, julho 2019. 
ESTUDOS LINGUÍSTICOS (SÃO PAULO. 1978)

GRUPO DE ESTUDOS LINGUÍSTICOS DO ESTADO DE SÃO PAULO (GEL)

Universidade Estadual Paulista "Júlio de Mesquita Filho" (UNESP) Instituto de Biociências, Letras e Ciências Exatas (IBILCE)

Rua Cristóvão Colombo, 2265 - CEP: 15054-000 Jardim Nazareth - São José do Rio Preto - SP - Brasil

http://www.gel.org.br/estudoslinguisticos/ estudoslinguisticos@gel.org.br

\section{Diretoria do GEL (Gestão UFSCar - 2019-2021)}

(Presidente) Luiz André Neves de Britto, Universidade Federal de São Carlos (UFSCar), São Carlos, São Paulo, Brasil

(Vice-Presidenta) Mariana Luz Pessoa de Barros, Universidade Federal de São Carlos (UFSCar), São Carlos, São Paulo, Brasil

(Secretário) Renato Miguel Basso, Universidade Federal de São Carlos (UFSCar), São Carlos, São Paulo, Brasil

(Tesoureira) Rosa Yokota, Universidade Federal de São Carlos (UFSCar), São Carlos, São Paulo, Brasil

\section{Editora responsável}

Profa. Dra. Claudia Zavaglia, Universidade Estadual Paulista "Júlio de Mesquita Filho"

(UNESP), São José do Rio Preto, São Paulo, Brasil

\section{Comissão editorial}

Prof. Dr. Carlos Eduardo Mendes de Moraes, Universidade Estadual Paulista "Júlio de Mesquita Filho" (UNESP), Assis, São Paulo, Brasil

Prof. Dr. Marcelo Módolo, Universidade de São Paulo (USP), São Paulo, São Paulo, Brasil

Prof. Dr. Oto Araújo Vale, Universidade Federal de São Carlos (UFSCar), São Carlos, São Paulo, Brasil

Profa. Dra. Luciani Ester Tenani, Universidade Estadual Paulista "Júlio de Mesquita Filho" (UNESP), São José do Rio Preto, São Paulo, Brasil

Profa. Dra. Maria Irma Hadler Coudry, Universidade Estadual de Campinas (UNICAMP), Campinas, São Paulo, Brasil

Profa. Dra. Angela Cecília de Souza Rodrigues, Universidade de São Paulo (USP), São Paulo, São Paulo, Brasil

Profa. Dra. Beth Brait, Pontifícia Universidade Católica de São Paulo (PUC-SP), São Paulo, São Paulo, Brasil 


\section{Conselho editorial}

Prof. Dr. Ana Luisa Verani Leal, Universidade de Macau, Macau, China Prof. Dr. Bertrand Daunay, Universidade de Lille, Lille, França

Prof. Dr. Eric Laporte, Université Paris-Est Marne-la-Vallée, Champs-sur-Marne, França

Prof. Dr. Frantome Bezerra Pacheco, Universidade Federal do Amazonas (UFAM), Manaus, Amazonas, Brasil

Profa. Dra. Inmaculada Penadés Martinez, Universidad de Alcalá (UAH), Madrid, Espanha

Profa. Dra. Julia Sevilla Muñoz, Universidad Complutense de Madrid, Madrid, Espanha Profa. Dra. Lou-Ann Kleppa, Universidade Federal de Rondônia (UNIR), Porto Velho, Rondônia, Brasil

Profa. Dra. Luisa A. Messina Fajardo, Università di Roma Tre, Roma, Itália Prof. Dr. Marcos Lopes, Universidade de São Paulo (USP), São Paulo, São Paulo, Brasil Profa. Dra. Maria Luisa Ortiz Alvarez, Universidade de Brasília (UnB), Brasília, Distrito Federal, Brasil

Profa. Dra. Renira Rampazzo Gambarato, Jönköping University, Jönköping, Sweden

Prof. Dr. Roberto Francavilla, Università degli Studi di Genova, Genova, Itália

Prof. Dr. Roberto Gomes Camacho, Universidade Estadual Paulista "Júlio de Mesquita Filho" (UNESP), São José do Rio Preto, São Paulo, Brasil

Prof. Dr. Ronaldo Lima Junior, Universidade Federal do Ceará (UFC), Fortaleza, Ceará, Brasil Profa. Dra. Sabela Fernández-Silva, Universidad Católica de Valparaíso (UCV), Valparaíso, Chile

Prof. Dr. Salvio Martín Menéndez, Universidad de Buenos Aires (UBA), Buenos Aires, Argentina

Prof. Dr. Sirio Possenti, Universidade Estadual de Campinas (UNICAMP), Campinas, São Paulo, Brasil

Profa. Dra. Tânia Romero, Universidade Federal de Lavras (UFLa), Lavras, Minas Gerais, Brasil

Prof. Dr. Tony Berber Sardinha, Pontifícia Universidade Católica de São Paulo (PUC-SP), São Paulo, São Paulo, Brasil

\section{Auxiliar editorial}

Milton Bortoleto, Universidade de São Paulo (USP), São Paulo, São Paulo, Brasil

\section{Revisão, normatização, projeto gráfico e diagramação}




\section{Catalogação na Publicação elaborada por}

Gildenir Carolino Santos (CRB-8 $\left.{ }^{\mathrm{a}} / 5447\right)$

Estudos Linguísticos (São Paulo. 1978). - v.1, n.1 (1978-). - São José do Rio Preto, SP: Grupo de Estudos Linguísticos do Estado de São Paulo, 20021 recurso digital : il.

Periodicidade quadrimestral desde volume 36, 2007 (atual).

Periodicidade anual até volume 35, 2006.

Periodicidade semestral até volume 29, 2000.

ISSN 1413-0939 (impresso).

Publicada no formato impresso até volume 29, 2000

Publicada no formato em CD-ROM dos volumes 30 ao 35 (2001 a 2006).

Disponível online a partir do volume 36, 2007.

Título abreviado: Est. Ling.

Preservada digitalmente no LOCKSS.

Disponível em: https://revistas.gel.org.br/estudos-linguisticos

1. Estudos linguísticos - Periódicos. 2. Linguística - Periódicos. I. Grupo de Estudos Linguísticos do Estado de São Paulo. 


\section{SUMÁRIO}

Apresentação

589

Claudia Zavaglia

Denominações para a brincadeira "amarelinha" no

estado de São Paulo: análise diatópica e léxicosemântica

Beatriz Aparecida Alencar

A polêmica sobre a imigração no Brasil: questões de memória discursiva

Patrícia Aparecida de Aquino e Daiane Rodrigues de Oliveira Bitencourt

Aspectos semânticos dos verbos cognitivos deduzir

e calcular no português

Letícia de Almeida Barbosa-Santos e Solange de Carvalho Fortilli

Letramento crítico e ensino de espanhol como língua estrangeira: questões

teórico-metodológicas no contexto dos Institutos Federais (IFs)

Larissa Cristina Arruda de Oliveira Benedini

Dicionário e locuções: uma proposta bilíngue (português-italiano)

Fábio Henrique de Carvalho Bertonha e Claudia Zavaglia

Aspectos prosódicos das sentenças interrogativas globais do português de

São Tomé: uma análise inicial

Gabriela Braga

Transformações econômicas e mudança linguística:

a língua em Louveira/SP

Victor Carreão

Dilma Rousseff e o ethos de amável coragem: o corpo no feminino 
Interfaces no processo de mediação em teletandem português e espanhol:

o papel dos mediadores

Kelly Cristiane Henschel Pobbe de Carvalho

e Karin Adriane Henschel Pobbe Ramos

O pré-teste ao EPPLE (Exame de Proficiência para Professores de Línguas

Estrangeiras): validade e desafios tecnológicos

Jéssica Nunes Caldeira Cunha

Numerais na língua Mehináku (Arawak)

786

Paulo Henrique Pereira Silva de Felipe

Alçamento vocálico das vogais médias pretônicas

800

iniciais na variedade do noroeste paulista

Márcia Cristina do Carmo

Diário escrito para a posteridade: os diários de Getúlio Vargas

Nathalia Reis Fernandes

A forma perifrástica ainda mais: uma descrição discursivo-funcional

Michel Gustavo Fontes

As ficções de fã e o Ensino de Línguas por Tarefas

860

William Danilo Garcia

A função de marcador aspectual de sintagmas adverbiais nas construções participiais absolutas licenciadas em textos de autores portugueses dos séculos XV, XVI e XVII

Alba Verôna Brito Gibrail

A crença nas palavras: (des)construções lexicais em antropônimos de líderes religiosos

Carlos Alexandre Gonçalves

Memes no meio digital: um olhar teórico sobre sua

Sergio Mikio Kobayashi 
A utilização de corpora em atividades de língua inglesa voltadas para a compreensão escrita de vestibulandos

Luana Aparecida Nazzi Laranja

Linguagem e interação: o ensino/aprendizagem de Língua Portuguesa por

meio da reestruturação textual na formação do sujeito leitor/autor

Marta Luzzi

O emprego de testes de factualidade em advérbios modais

George Henrique Nagamura

Uma análise sobre as categorias de desumanização do sujeito entre as personagens negras na obra Úrsula, de Maria Firmina dos Reis

José Gomes Pereira

A realização variável da segunda pessoa por bilíngues em vêneto $e$ português em uma comunidade rural do Espírito Santo

Edenize Ponzo Peres e Maria do Socorro Vieira Coelho

A moça tecelã: um texto instigante

1030

Nelyse A. Melro Salzedas e Rivaldo Alfredo Paccola

A descentralização do sujeito lírico em Micheliny Verunschk

1044

Édila de Cássia Souza Santana

Eventos tonais pré-nucleares de declarativas e interrogativas neutras do português do Libolo em fala semiespontânea

Vinícius Gonçalves dos Santos e Flaviane Romani Fernandes Svartman

Entre a ciência e a diversão: análise comparativa de

enunciados audiovisuais de divulgação científica e

suas formas de diálogo com o público presumido

Beatriz Amorim de Azevedo e Silva

Percepção auditiva de fronteiras de palavras e de clíticos fonológicos no Português Brasileiro

Lilian Maria da Silva 
O desenvolvimento da negação: proposta de classificação funcional das construções negativas em desenvolvimento

Angelina Nunes de Vasconcelos

Argumentação, humor e incongruência na linguagem da criança

Alessandra Jacqueline Vieira e Alessandra Del Ré 